\title{
Morphological canalization, integration and plasticity in response to population density in Abutilon theophrasti: influences of soil conditions and growth stages
}

\author{
Shu Wang ${ }^{1}$ and Dao-Wei Zhou ${ }^{2}$ \\ ${ }^{1}$ Guizhou University \\ ${ }^{2}$ Northeast Institute of Geology and Agroecology, Chinese Academy of Sciences
}

June 30, 2021

\begin{abstract}
Phenotypic integration and developmental canalization have been hypothesized to constrain the degree of phenotypic plasticity, but there is little evidence for the relationships among the three processes in different environments, especially for plants under natural conditions. To address this issue, we conducted a field experiment by subjecting plants of Abutilon theophrasti to low, medium and high densities, under infertile and fertile soil conditions, measured a variety of traits and analyzed canalization (coefficient of variation [CV]), integration (coefficient of integration [CI] and the number of significant correlations of a trait with other traits $[\mathrm{NC}]$ ), and plasticity (REL RDPIs and ABS RDPIs) in these traits and their relationships at two stages of plant growth. Our results showed an increase in mean CV, NC and ABS RDPIs of traits with density, and the positive correlations between trait NC and ABS RDPIs became stronger with higher densities but weaker over time in fertile soil, while correlations among trait CV, NC and ABS RDPIs became stronger over time in infertile soil. Results suggested shared or cooperation mechanisms among phenotypic integration, canalization and plasticity. Soil conditions and growth stage may affect responses of these correlations to density via modifying plant size and competition strength. The attenuated canalization and enhanced integration may be helpful for the production of plasticity, especially under intense competition.
\end{abstract}

\section{Introduction}

Phenotypic plasticity, defined as the ability to produce different phenotypes in response to different environmental conditions (Bradshaw, 1965; Massimo Pigliucci, 2005). The expression of a phenotype under a certain environment is the result of the integration of local responses of many modules or traits and their interactions (Pigliucci \& Preston, 2004). Consequently, the evolution of a given trait and its plasticity may be subject to restrictions due to its genetic correlations with other traits (Agrawal \& Stinchcombe, 2009; Gianoli \& Palacio-López, 2009). Not all species or traits are plastic, probably because the extent of phenotypic plasticity is limited by both intrinsic and extrinsic factors (DeWitt et al., 1998; Givnish, 2002; Valladares et al ., 2007).

A process that may limit phenotypic plasticity is developmental canalization, which is generally assessed by coefficient of variation (CV) in traits (Woods et al. , 1999). Developmental canalization, or robustness, is the property of an organism that buffers development against environmental and genetic perturbations to produce a consistent phenotype (Waddington, 1957), including environmental canalization and genetic canalization (Wagner et al. , 1997). Environmental canalization indicates the insensitivity of traits to environmental perturbations in variable environments (Debat \& David, 2001; S.C. Stearns et al., 1995; Wagner et al. , 1997), like a process in opposition to phenotypic plasticity (Stearns et al. , 1995; Wilkins, 1997). But both phenotypic plasticity and environmental canalization may reflect the ability of an organism to adjust phenotypic expression appropriately in dealing with environmental changes, at individual and population 
levels respectively (Reed et al. , 2010; Schlichting \& Pigliucci, 1998). Genetic canalization, refers to the buffering of the effects of genetic variation (Wagner et al. , 1997). The developmental canalization here may include both genetic and environmental canalization. It is widely appreciated that stress conditions increase phenotypic variability in traits (Woods et al. , 1999). If both plasticity and instability of traits increase with environmental stress, it is reasonable to infer some common mechanisms in charge of the two kinds of variabilities (Meiklejohn \& Hartl, 2002). Wagner et al.(1997) hypothesized that selection for environmental canalization may facilitate the evolution of genetic canalization, given the ample evidence for genetic canalization from empirical studies (Dun \& Fraser, 1958; Dworkin, 2005; Polaczyk et al. , 1998; but see Hermisson \& Wagner, 2004). However, relevant studies did not find changes of environmental canalization affect the level of genetic canalization (Debat et al. , 2009; Ian Dworkin, 2005; Meiklejohn \& Hartl, 2002). In the unpredictable environments of Mediterranean ecosystem, environmental stress favors both plasticity and high degree of genetic variations across species in oak seedlings (Valladares et al., 2002), implying negative correlations between plasticity and canalization. Studies on the relationships between trait plasticity and canalization are rarely seen, even less on how environments affect their relationship, especially for plant species.

Phenotypic integration may also play a role as an internal constraint to phenotypic plasticity (Gianoli, 2001, 2003; Pigliucci et al. , 1995; Schlichting, 1986, 1989; Valladares et al. , 2007). Phenotypic integration refers to the pattern and magnitude of character correlations (M. Pigliucci \& Preston, 2004), which results from genetic, developmental and/or functional connections among traits (Pigliucci \& Preston, 2004; Schlichting \& Pigliucci, 1998), and is often expressed in terms of the number of significant phenotypic correlations between traits (Pigliucci, 2002; Pigliucci \& Marlow, 2001; Schlichting, 1989). Evidence has shown that the degree of plasticity in response to shading or drought in a given trait decreased with the increase of the number of its correlations with other traits in two local species from Chile, suggesting the restriction of phenotypic integration to the degree of plasticity (Gianoli \& Palacio-López, 2009), and the greater the constraints of genetic correlations for a trait, the lower its ability to respond to environments. On the other hand, however, the strength of phenotypic integration may also increase with environmental stresses (García-Verdugo et al. , 2009; Gianoli, 2004; Schlichting, 1989; Waitt \& Levin, 1993), which also induce plastic responses in traits, implying the positive correlations between phenotypic integration and plasticity in traits. Since most empirical studies have only studied patterns of phenotypic integration in a single environment (Pigliucci \& Preston, 2004; but see Liu et al. , 2007; Pigliucci et al. , 1995), we still know surprisingly little about how the environment influences levels of phenotypic integration (Mallitt et al. , 2010), even less on the relationship between phenotypic integration and plasticity and its variation with environmental conditions.

Population density, as one of the major natural stresses that result in size variations in plants, its variation can result in the heterogeneity of multiple environmental factors, inducing complex responses in traits. Both variation among individuals and phenotypic plasticity can impart integration among morphological traits (Klingenberg, 2014). Thereby such responses to density may also correlate with developmental canalization and phenotypic integration. Correlations can vary with different abiotic conditions and growth stages, due to their influences on trait plasticity (Wang et al. , 2017), integration and canalization (Damiánet al. , 2018; Goswami et al ., 2015). We need more detailed studies on integration, canalization and plasticity to generalize about the relationships among them (Kavanagh, 2020), especially at different stages of plant growth or under different abiotic conditions. As traits may differ in the magnitude of their plastic responses to different resources, resulting in a "hierarchy" of responses (White, 1979), the importance of considering a wider range of plant traits is underscored (Ryser \& Eek, 2000). Here we conducted a field experiment on an annual herbaceous species of Abutilon theophrasti, to analyze phenotypic plasticity, canalization and integration for a number of allocation and morphological traits and their relationships in response to density for plants under two contrasting soil conditions at two growth stages. We aimed to test the following hypotheses: 1) trait plasticity, canalization and integration increase with higher densities; 2) there are positive correlations among the three processes, which intensified with greater densities; 3) soil conditions and growth stage can influence responses of their correlations to density.

\section{Materials and methods}




\section{Study species}

Abutilon theophrasti Medicus (Malvaceae) is an annual weedy species, native to China and India. It usually grows to a height of up to $1-1.5 \mathrm{~m}$, and can reach reproductive maturity within $90 \mathrm{~d}$, completing its life cycles in $\sim 5$ mo (McConnaughay \& Coleman, 1999), with substantial plasticity in allocation, morphology and architecture in response to varying environmental factors (McConnaughay \& Bazzaz, 1992). It colonizes relatively nutrient-rich habitats and is typically found in open fields, on roadsides, and in gardens.

\section{Experimental design}

The experiment was conducted in 2007 at the Pasture Ecological Research Station of Northeast Normal University, Changling, Jilin province, China ( $44^{\circ} 45^{\prime} \mathrm{N}, 123^{\circ} 45^{\prime} \mathrm{E}$ ). Seeds of $A$. therophrasti were collected from local wild populations near the research station in the late August of 2006 and were dry stored at $-4^{\circ} \mathrm{C}$. We used a split plot design, with soil conditions as the main factor, density and block as a sub-factor. Two large plots were assigned as two (infertile and fertile) soil conditions, each was divided into nine $2 \times 3 \mathrm{~m}$ sub-plots and randomly arranged with three treatments of densities and blocks. Seeds of $A$. theophrasti were sown on June 7, 2007, with three inter-planting distances of 30,20 and $10 \mathrm{~cm}$, to reach target plant densities of 13.4, 36 and 121 plants per $\mathrm{m}^{-2}$, assigned as Low, Medium and High density treatments respectively. Most seeds emerged $4 \mathrm{~d}$ after sowing. Seedlings were thinned to the target densities at four-leaf stage. Plots were hand-weeded when necessary and watered regularly.

We established the infertile soil conditions as a plot using the original soil of experimental field at the station that had been used annually for many years (aeolian sandy soil). The fertile soil conditions was set up by covering the other large plot with $5-10 \mathrm{~cm}$ virgin soil transported from a nearby meadow with no cultivation history (meadow soil), with contrasting nutrient contents of the two soil conditions (Wang et al., 2017). The meadow soil is not located far away from the experiment field, which used to be meadow as well and has been reclaimed for experimental use since the establishment of research station. Therefore, basically the soil of the experimental field was the same type as the meadow soil, but with different conditions or qualities. Covering the other plot with meadow soil led to a greater amount of soil or nutrients for the fertile soil treatment, which also led to thicker soil layers of the fertile plot than the infertile one. To keep the soil and resource amounts as even as possible, we crushed the blocky soil into very small bits, and mingled them adequately, before spreading them over the entire plot and compaction. Seeds were sown into all plots at the same burial depth and sowing rate.

\section{Data collection}

Plants were harvested at 30, 50 and $70 \mathrm{~d}$ of plant growth, representing developmental stages of early vegetative growth, late vegetative or early reproductive growth, and middle to late reproductive growth respectively. At each stage, six individual plants were randomly chosen from each plot, making a total of 6 replicates $\times 3$ plots $\times 3$ densities $\times 2$ soils $\times 3$ stages $=324$ samplings. For each plant, the following traits were measured if applicable: the length of stem, diameter at the basal of stem, petiole length and angle, leaf number, lamina width (lamina size), branch length, angle and number, main root length, main root diameter, lateral root length and lateral root number (above or equal to $1 \mathrm{~mm}$ in diameter along the main root). Morphological traits of plants at $30 \mathrm{~d}$ of growth were not taken into account due to small plant sizes. Each individual plant was then separated into root, stem, petiole, leaf, reproductive and branch parts if any, oven-dried at $75^{\circ} \mathrm{C}$ for two days and weighed.

\section{Statistical analysis}

All statistical analyses were conducted using SAS statistical software (SAS Institute 9.0 Inc. 2002). All traits were used in analyses (abbreviations see Table 1). All data were log-transformed except for petiole angles and branch angles (square root-transformed) to minimize variance heterogeneity before statistical analysis. Three-way ANOVA and ANCOVA were performed to evaluate the overall effects of growth stage, soil condition and population density and their interactions on all traits, with total mass nested in growth stage as a covariate in three-way ANCOVA. Within each soil condition at each stage, effects of density were 
analyzed by one-way ANOVA for total mass, and one-way ANCOVA for all the other traits with total mass as a covariate. Adjusted mean values of traits produced from multiple comparisons by LSD method of the General Linear Model (GLM) program in ANCOVAs were used in calculation of plasticity.

The plasticity in traits was evaluated with the revised simplified Relative Distance Plasticity Index (RDPI $)_{\mathrm{s}}$, for its strong statistical power in tests of differences in plasticity (F. Valladares, Sanchez-Gomez, \& Zavala, 2006). For a given trait, its $\mathrm{RDPI}_{\mathrm{S}}$ values in response to high and medium densities relative to low density $\left(\mathrm{H}-\mathrm{L} \mathrm{RDPI}_{\mathrm{S}}\right.$ and $\left.\mathrm{M}-\mathrm{L} \mathrm{RDPI}_{\mathrm{s}}\right)$ were calculated as:

$\mathrm{RDPI}_{\mathrm{s}}=(X-Y) /(X+Y)$

where $X$ was the adjusted mean trait value at high or medium density, and $Y$ was the mean value at low density. Both the level and degree of plasticity (relative plasticity and absolute plasticity) in traits were calculated as REL RDPI and ABS RDPI $I_{\mathrm{s}}$ respectively.

Phenotypic canalization was evaluated by coefficient of variation (CV) for a given trait, calculated as the standard deviation divided by mean value of the trait. Phenotypic integration was estimated with the number of significant correlations of a trait with other traits $(\mathrm{NC} ; \mathrm{p}<0.05)$ and coefficient of integration $(\mathrm{CI})$ (Cheverud, Rutledge, \& Atchley, 1983). Correlations among traits were evaluated by Pearson Correlation Coefficients (PCC) produced by PROC CORR (Gianoli \& Palacio-López, 2009). CI for traits was computed as:

$I=\left[[?](\lambda-1)^{2} /\left(n^{2}-n\right)\right]^{1 / 2}$

where $\mathrm{n}$ is the number of traits and $\lambda$ is an eigenvalue of the correlation matrix of the normalized data.

Both Correlation and regression analyses were applied to qualify and quantify the relationships between phenotypic plasticity $\left(\mathrm{RDPI}_{\mathrm{s}}\right)$ and phenotypic canalization $(\mathrm{CV})$ or integration $(\mathrm{NC})$ at different densities for plants in each soil conditions at each stage. Results of correlations and regressions were also analyzed with three-way ANOVA to access effects of population density, soil conditions and growth stage and their interactions; and one-way ANOVA for effects of density on these relationships in each soil conditions at each stage.

\section{Results}

Effects of stage, soil, density, and interaction between stage and soil were significant for most traits, and interactions between stage and density, between soil and density were also significant for several different traits (Table S1). Plant size (total mass) explained significant variations in most traits. Across both stages, infertile vs. fertile soil and high and medium vs. low density reduced total biomass $(P<0.001$; Fig. S1).

Responses of variables to density

Density had significant effects on mean coefficient of variation (CV), mean number of correlations (NC), and mean degree of plasticity $\left(\mathrm{ABS}_{\mathrm{RDPI}}\right)$ for all traits $(P<0.05$; Table 2$)$. However, responses of variables to density became less significant over time (Fig. 1). Across both soil conditions at $50 \mathrm{~d}$, compared to low density, high and medium densities increased CI (LSD, $P<0.010$ ). In fertile soil, high vs low density increased mean CV by $20 \%$ at $50 \mathrm{~d}(P=0.046)$, decreased NC slightly at $70 \mathrm{~d}(P=0.067)$. No difference in mean CV and CI between densities was found at $70 \mathrm{~d}$. CV values were significant in more traits, NC values tended to decrease, with higher densities in fertile soil (Table S2, S3). Across both growth stages, mean ABS RDPI $\mathrm{S}_{\mathrm{S}}$ in response to high density was greater than that in response to medium density, compared to low density in infertile soil $(P<0.001$; Fig. 1$)$. Total mass was highly plastic, with the average relative plasticity $(\text { REL RDPI })_{\mathrm{s}}$ ) of -0.433 in response to high density across all soil conditions and stages and other traits had plasticity varying with soil conditions and growth stages (Table S4).

\section{Relationships among variables}

Trait NC had negative correlations with REL RDPI , and positive correlations with ABS RDPI , and trait CV mainly had positive correlations with RDPI $_{\mathrm{S}}$ (Fig. 2 and Table S5). These correlations decreased over 
time in fertile soil, and increased over time in infertile soil. Soil conditions, growth stage and population

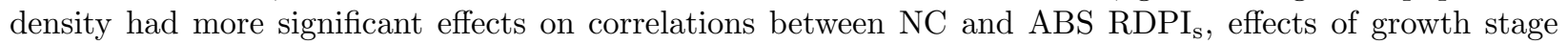
and population density were more significant for correlations between CV and REL RDPI (Table 3). For both stages, density effects on correlations between $\mathrm{NC}$ and $\mathrm{RDPI}_{\mathrm{s}}$ and between $\mathrm{CV}$ and $\mathrm{RDPI}_{\mathrm{s}}$ were more significant in fertile vs. infertile soil (Table 4 and Fig. 2).

\section{Discussion}

\section{Developmental canalization}

Theoretically, it is predicted increments in environmental variations may lead to relatively higher levels of plasticity in morphological traits (Bradshaw, 1965; de Jong, 1995), whereas fitness-traits may be more likely to maintain stable under a range of environmental conditions (Lerner, 1954; Stearns \& Kawecki, 1994; Waddington, 1957; Wagner et al., 1997). Characters more closely related to fitness are expected to be better buffered against environmental effects because deviations from the optimal phenotype will be strongly selected against (Clarke, 1995; Lerner, 1954; Stearns \& Kawecki, 1994; Waddington, 1957). These predictions have been proved by the contrasting performances in the degree of plasticity in response to temperature between morphological traits and fitness traits (Liefting et al. , 2009). However, we did not find reproductive mass ratio had lower plasticity in response to density than other traits, and its amongindividual variation $(\mathrm{CV})$ was higher than other traits at different densities, consistent with other studies (Woodset al. , 1999). It suggested the relative stability of fitness traits depends on the level or range of environmental variations, and increased phenotypic variations can be produced under highly stressful conditions that plants are incapable to adapt (Woods et al. , 1999). When the stress is severe enough, the buffering against drastic changes may no longer be able to prevent such overt changes, and phenotypic variability in more robust traits might assist survival at the population level (Elgart et al. , 2015). The increase of density should have been severe enough to cause the decrease of reproductive allocation in this study.

\section{Phenotypic integration}

In spite of the recognized importance that changes in the correlation structure can have for evolutionary change (Lande \& Arnold, 1983), we still know surprisingly little about how the environment influences levels of phenotypic integration. Despite much recent progress on this topic (Pigliucci \& Preston, 2004; Schlosser \& Wagner, 2004), most empirical studies have only studied patterns of phenotypic integration in a single environment (Pigliucci \& Preston, 2004; but see Liu et al., 2007; Pigliucci et al. , 1995). In this study, we found an increase in coefficient of integration (CI) with density at $50 \mathrm{~d}$ (Gianoli, 2004; Schlichting, 1986; Wylde \& Bonduriansky, 2020), and decrease in the number of correlations (NC) with density at 70 d (Badyaev et al. , 2005; Mallitt et al. , 2010; Pigliucci \& Kolodynska, 2002). The stage-dependence responses in CI suggested the strength of response of integration to density decreased over time. It is hypothesized that the increase in the number and strength of correlations among functionally correlated traits (phenotypic integration) is related to the extent of environmental stress (Gianoli, 2004; Schlichting, 1986), and endow plants the ability to effectively respond to such stress (Chapin III, 1991). At $70 \mathrm{~d}$, density effects on plants became attenuated, due to small individuals being obsoleted (Wang et al. , 2017). This may lead to weakened responses in trait correlations. It is reported phenotypic integration contributed to differences between native and invasive species less than phenotypic plasticity (Osunkoya et al. , 2014), but differences in phenotypic integration plasticity were more distinguishable than differences in plasticity for clones ofDaPhnia magna (Plaistow \& Collin, 2014). It suggested phenotypic integration plays a more important role in plant adaptation to environmental stresses.

\section{Correlations among canalization, integration and plasticity}

Our results showed an increase in among-individual variations (mean coefficient of variation, CV) for all traits by high vs. low density in fertile soil only, indicating a decreased level of canalization in traits, consistent with other results (Imasheva, Loeschcke et al. , 1997; Ramler et al. , 2014; Teder et al. , 2008; Woods et al. , 1999). At $50 \mathrm{~d}$, plants in infertile soil were smaller, and displayed stronger responses to density than in 
fertile soil (Wanget al. , 2017). The decrease in canalization or increase in among-individual variations might be complementary to the increase in plasticity. However, there were also more positive correlations between the degree of plasticity (ABS RDPI $)_{s}$ ) and CV with higher densities, suggesting some shared mechanisms of the two process in response to environmental stress (Debat \& David, 2001; Meiklejohn \& Hartl, 2002). This was consistent with that plasticity and within-environment variation are favored by environmental stresses in the unpredictable environments of Mediterranean ecosystem (Valladares et al. , 2002). As sources of phenotypic variation, canalization and plasticity are hypothesized to under the control of some common mechanisms (Debat \& David, 2001; Meiklejohn \& Hartl, 2002), but proved by little evidence (Debat et al. , 2009; Dworkin, 2005). Phenotypic plasticity actually depicts an ability of an organism to react to external environmental changes via self-regulation, rather than simply a phenomenon of phenotypic difference or change. To the opposite, developmental canalization is a mechanism of an organism in buffering against environmental or genetic disturbances to minimize phenotypic variations (Waddington, 1952, 1956). The correlations between the two processes were not significant at low density, indicating both the adaptability to a novel environment and the robustness are compatible when a certain degree of phenotypic fluctuations exists due to noise (Kaneko, 2012). However, the presence of environmental stresses may favor stronger plasticity over canalization in traits. It seems the loss of canalization in some circumstances may not necessarily be harmful, but facilitate the production of plasticity.

In spite of a decrease in mean NC by high density at $70 \mathrm{~d}$, it had positive correlations with the magnitude of trait plasticity $\left(\mathrm{ABS} \mathrm{RDPI}_{\mathrm{s}}\right.$ ), which intensified with higher densities. It suggested in the evolution of traits within an individual organism, the traits of greater integration were more able to respond to environmental stress flexibly, or traits of greater plasticity are more likely affect the variations of other traits. The mechanisms behind differences of organismal systems in their capacity to buffer or accommodate stress-induced variation are poorly understood (Badyaev et al., 2005). On the one hand, buffering of a stressor might be a consequence of developmental complexity rather than an evolved resistance mechanism for resilience to stressors (Meiklejohn \& Hartl, 2002; Rice, 1998; Siegal \& Bergman, 2002; Waxman \& Peck, 1998). Under this scenario, the complexes of traits that share the greatest number of developmental interactions (i.e., the most developmentally integrated) should be the most able to maintain functionality and to accommodate the effects of stress during ontogeny. On the other hand, an organism's ability to function in different environments requires the ability to track and respond to environmental change (Eshel \& Matessi, 1998; Carl D. Schlichting \& Smith, 2002; Waddington, 1941; Wagner et al. , 1997). The traits of stronger integration have more connections with other traits, thus are more able to dominate the phenotypic variation, and enhance the whole-plant fitness through plastic responses. Because integration can alleviate the constraints to trait plasticity by environmental signal amplification or inhibition through developmental interaction among trait plasticity (Lande, 2019). Inversely, traits of greater plasticity are more likely to affect other traits through phenotypic integration, leading to stronger correlations among traits.

Both phenotypic integration and among-individual variation positively correlated with the degree of plasticity, suggesting some cooperation mechanisms among the three processes. Greater flexibility of individual systems is hypothesized to be produced by lessening their homeostatic integration (West-Eberhard, 2003). Such a decrease might enhance the range of performance of individual organismal systems and ultimately increase organismal capacity to adapt to changing conditions (Badyaev et al. , 2005; Rutherford, 2003). Meanwhile, mechanisms for phenotypic plasticity and developmental canalization may be always functioning in an organism. Organisms can maintain functionality in stressful environments by channeling stress-induced developmental variation through buffering some organismal functions while increasing the flexibility of others (Alberch, 1980; Nijhout, 2002). And the relaxation of canalization to some extent may assist the production of plasticity in traits.

\section{Effects of soil conditions and growth stage}

Correlations among trait canalization, integration and plasticity not only varied with greater densities, but such variations also change with soil conditions and growth stages. (Goswami et al. , 2015) showed an increase in correlation between fluctuating asymmetry and integration over time. But we found correlations 
among integration, canalization and plasticity increased over time in infertile soil, and decreased over time in fertile soil. As competition intensity may increase over time in infertile soil, and decrease over time in fertile soil (Wang et al. , 2017), soil condition and growth stage may also affect these correlations through modifying plant size and competition intensity. Additionally, since the positive correlations between integration and plasticity became stronger with higher densities in fertile soil only, it suggested plants in favorable conditions are more able to deal with the increase of density via coordination of trait plasticity and integration, whereas unfavorable conditions may attenuate this ability.

\section{Conclusions}

Our results showed an increase in phenotypic integration, plasticity and among-individual variation with density, and the positive correlations between integration and plasticity became stronger with higher densities but weaker over time in fertile soil, while the correlations among the three processes became stronger over time in infertile soil. These suggested certain shared or cooperation mechanisms for integration, canalization and plasticity in traits. Soil conditions and growth stage may affect the responses to density in correlations among the three processes via effects on plant size and competition intensity. Phenotypic integration may play a more important role in plant adaptation to environmental stresses, and the decrease in canalization level may not be harmful. The released variations among individuals due to attenuated canalization and enhanced integration may facilitate plant reaction and adaptation to environmental stresses, and inversely plasticity may lead to higher level of phenotypic integration and reduced canalization, especially when plants experience intense competition.

\section{Supplementary Information}

The online version contains supplementary materials of Table S1 S5, Fig. S1.

\section{Acknowledgements}

We are grateful the reviewers and editors who all provided useful feedback on this manuscript. This work was funded by the National Natural Science Foundation of China (NSFC, 31800335), Guizhou Province Science and Technology Planning Program (2019-1089) and Guizhou University Talent Introduction Research Program (2017-39) to SW.

\section{Conflict of interest}

No conflict of interests have been declared.

\section{Author contributions}

Both authors contributed to the study conception and design. Material preparation, data collection and analysis were performed by Shu Wang. The draft of the manuscript was written and edited by Shu Wang. All authors read and approved the final manuscript.

\section{Data Accessibility}

- Sampling morphological data: Dryad doi: 10.5061/dryad.bvq83bk8c.

\section{References}

Agrawal, A. F., \& Stinchcombe, J. R. (2009). How much do genetic covariances alter the rate of adaptation? Proceedings of the Royal Society of London B: Biological Sciences, 276 (1659), 1183-1191.

Alberch, P. (1980). Ontogenesis and morphological diversification. American Zoologist, 20 , $653-667$.

Badyaev, A. V., Foresman, K. R., \& Young, R. L. (2005). Evolution of morphological integration: developmental accommodation of stress-induced variation. The American Naturalist, 166 (3), 382-395.

Bradshaw, A. D. (1965). Evolutionary significance of phenotypic plasticity. Advances in Genetics, 13 , $115-155$. 
Chapin III, F. S. (1991). Integrated responses of plants to stress. BioScience, 41 , 29-36.

Cheverud, J. M., Rutledge, J. J., \& Atchley, W. R. (1983). Quantitative genetics of development: genetic correlations among age-specific trait values and the evolution of ontogeny. Evolution, 37, 895-905.

Clarke, G. M. (1995). The genetlc basis of developmental stability. II. Asymmetry of extreme phenotypes revisited. . The American Naturalist, 146 , 708-725.

Damián, X., Fornoni, J., Domínguez, C. A., \& Boege, K. (2018). Ontogenetic changes in the phenotypic integration and modularity of leaf functional traits. Functional Ecology, 32 (2), 234-246.

De Jong, G. (1995). Phenotypic plasticity as a product of selection in a variable environment.American Naturalist, $145,493-512$.

Debat, V., \& David, P. (2001). Mapping phenotypes: canalization, plasticity and developmental stability. Trends in Ecology and Evolution, 16 (10), 555-561.

Debat, V., Debelle, A., \& Dworkin, I. (2009). Plasticity, canalization, and developmental stability of theDrosophila wing: Joint effects of mutations and developmental temperature. Evolution, 63 , 2864-2876.

DeWitt, T. J., Sih, A., \& Wilson, D. S. (1998). Costs and limits of phenotypic plasticity. Trends in Ecology and Evolution, 13 (2), 77-81.

Dun, R. B., \& Fraser, A. S. (1958). Selection for an invariant character-vibrissa number-in the house mouse. Nature, 181 , 1018-1019.

Dworkin, I. (2005). Evidence for canalization of Distal-less function in the leg of Drosophila melanogaster. Evolution 6 development, 7 , 89-100.

Dworkin, I. (2005). A study of canalization and developmental stability in the sternopleural bristal system of Drosophilla Melanogaster . Evolution, 59 , 1500-1509.

Elgart, M., Snir, O., \& Soen, Y. (2015). Stress-mediated tuning of developmental robustness and plasticity in flies. Biochimica et Biophysica Acta, 2015 , 462-466.

Eshel, I., \& Matessi, C. (1998). Canalization, genetic assimilation and preadaptation: a quantitative genetic model. Genetics, 149 (4), 2119-2133.

García-Verdugo, C., Granado-Yela, C., Manrique, E., Casas, R. R. d., \& Balaguer, L. (2009). Phenotypic plasticity and integration across the canopy of Olea euripeaesubsp. Guanchica (Oleaceae) in populations with different wind exposures. American Journal of Botany, 96 (8), 1454-1461.

Gianoli, E. (2001). Lack of differential plasticity to shading of internodes and petioles with growth habit in Convolvulus arvensis (Convolvulaceae).International Journal of Plant Sciences, 162 (6), 1247-1252.

Gianoli, E. (2003). Phenotypic responses of the twining vine Ipomoea purpurea (Convolvulaceae) to physical support availability in sun and shade. Plant Ecology, 165 (21-26).

Gianoli, E. (2004). Plasticity of traits and correlations in two populations of Convolvulus arvensis (Convolvulaceae) differing in environmental heterogeneity.International Journal of Plant Science, 165 , 825-832.

Gianoli, E., \& Palacio-López, K. (2009). Phenotypic integration may constrain phenotypic plasticity in plants. Oikos, 118 (12), 1924-1928.

Givnish, T. J. (2002). Ecological constraints on the evolution of plasticity in plants. Evolutionary Ecology, $16(3), 213-242$.

Goswami, A., Binder, W. J., Meachen, J., \& O'Keefe, F. R. (2015). The fossil record of phenotypic integration and modularity: a deep-time perspective on developmental and evolutionary dynamics. Proceedings of the National Academy of Sciences, 112 (16), 4891-4896. 
Hermisson, J., \& Wagner, G. P. (2004). The population genetic theory of hidden variation and genetic robustness. Genetics, 168, 2271-2284.

Imasheva, A. G., Loeschcke, V., Zhivotovsky, L. A., \& Lazebny, O. E. (1997). Effects of extreme temperatures on phenotypic variation and developmental stability inDrosophila melanogaster and Drosophila buzzatii .Biological Journal of the Linnean Society, 61 (1), 117-126.

Kaneko, K. (2012). Evolution of robustness and plasticity under environmental fluctuation: formulation in terms of phenotypic variances. Journal of Statistical Physics, 148, 687-705.

Kavanagh, K. (2020). Developmental plasticity associated with early structural integration and evolutionary patterns: Examples of developmental bias and developmental facilitation in the skeletal system. Evolution $\mathscr{E}$ development, 22 (1-2), 196-204.

Klingenberg, C. P. (2014). Studying morphological integration and modularity at multiple levels: concepts and analysis. Philosophical Transactions of the Royal Society B: Biological Sciences, 369 (1649), 20130249.

Lande, R. (2019). Developmental integration and evolution of labile plasticity in a complex quantitative character in a multiperiodic environment. Proceedings of the National Academy of Sciences, 116 (23), 1136111369 .

Lande, R., \& Arnold, S. J. (1983). The measurement of selection on correlated characters. Evolution, 37 (6), $1210-1226$.

Lerner, I. M. (1954). Genetic Homeostasis . New York: Wiley.

Liefting, M., Hoffmann, A. A., \& Ellers, J. (2009). Plasticity versus environmental canalization: population differences in thermal responses along a latitudinal gradient in Drosophila serrata . Evolution, 63 (8), 19541963.

Liu, Y., Schieving, F., Stuefer, J. F., \& Anten, N. P. R. (2007). The effects of mechanical stress and spectral shading on the growth and allocation of ten genotypes of a stoloniferous plant. Annals of Botany, 99 (1), 121-130.

Mallitt, K. L., Bonser, S. P., \& Hunt, J. (2010). The plasticity of phenotypic integration in response to light and water availability in the pepper grass, Lepidium bonariense . Evolutionary Ecology, 24, 1321-1337.

McConnaughay, K. D. M., \& Bazzaz, F. A. (1992). The occupation and fragmentation of space: consequences of neighbouring shoots. Functional Ecology, 6 , 711-718.

McConnaughay, K. D. M., \& Coleman, J. S. (1999). Biomass allocation in plants: ontogeny or optimality? A test along three resource gradients. Ecology, 80 (8), 2581-2593.

Meiklejohn, C. D., \& Hartl, D. L. (2002). A single mode of canalization. Trends in Ecology \& Evolution, 17 , 468-473.

Nijhout, H. F. (2002). The nature of robustness in development. BioEssays, 24 , 553-563.

Osunkoya, O. O., Boyne, R., \& Scharaschkin, T. (2014). Coordination and plasticity in leaf anatomical traits of invasive and native vine species. American Journal of Botany, 101 (9), 1423-1436.

Pigliucci, M. (2002). Touchy and bushy: Phenotypic plasticity and integration in response to wind stimulation in Arabidopsis thaliana. International Journal of Plant Sciences, 163 (3), 399-408.

Pigliucci, M. (2005). Evolution of phenotypic plasticity: where are we going now? Trends in Ecology and Evolution, 20 (9), 481-486.

Pigliucci, M., \& Kolodynska, A. (2002). Phenotypic plasticity and integration in response to flooded conditions in natural accessions of Arabidopsis thaliana (L.) Heynh (Brassicaceae). Annals of Botany, 90 (2), 199-207. 
Pigliucci, M., \& Marlow, E. T. (2001). Differentiation for flowering time and phenotypic integration in Arabidopsis thaliana in response to season length and vernalization. Oecologia, 127 , 501-508.

Pigliucci, M., \& Preston, K. (2004).Phenotypic integration: studying the ecology and evolution of complex phenotypes. Oxford: Oxford Univeresity Press.

Pigliucci, M., Whitton, J., \& Schlichting, C. (1995). Reaction norms of Arabidopsis. I. Plasticity of characters and correlations across water, nutrient and light gradients. Journal of Evolution Biolology, 8 , 421-438.

Plaistow, S. J., \& Collin, H. (2014). Phenotypic integration plasticity in Daphnia magna : an integral facet of $\mathrm{G} \times$ E interactions. Journal of Evolutionary Biology, 27 (9), 1913-1920.

Polaczyk, P. J., Gasperini, R., \& Gibson, G. (1998). Naturally occurring genetic variation affectsDrosophila photoreceptor determination. Development, Genetics and Evolution, 207 , 462-470.

Ramler, D., Mitteroecher, P., Shama, L. N. S., Wegner, K. M., \& Ahnelt, H. (2014). Nonlinear effects of temperature on body form and developmental canalization in the threespine stickleback. Journal of Evolution Biolology, 27, 497-507.

Reed, T. E., Waples, R. S., Schindler, D. E., Hard, J. J., \& Kinnison, M. T. (2010). Phenotypic plasticity and population viability: the importance of environmental predictability. Proceedings of the Royal Society B-Biological Sciences, 277, 3391-3400.

Rice, S. H. (1998). Evolution of canalization and the breaking of von Baer's laws: modeling the evolution of development with epistasis. Evolution, 52, 647-656.

Rutherford, S. L. (2003). Between genotype and phenotype: protein chaperones and evolvability. Nature Reviews Genetics, 4, 263-274.

Ryser, P., \& Eek, L. (2000). Consequences of phenotypic plasticity vs. interspecific differences in leaf and root traits for acquisition of aboveground and belowground resources. American Journal of Botany, 87 (3), 402-411.

Schlichting, C. D. (1986). The evolution of phenotypic plasticity in plants. Annual Review of Ecology and Systematics, 17 (1), 667-693.

Schlichting, C. D. (1989). Phenotypic integration and environmental change. BioScience, 39 , 460-464.

Schlichting, C. D. (1989). Phenotypic plasticity in Phlox II. Plasticity of character correlations. Oecologia 78 , 496-501.

Schlichting, C. D., \& Pigliucci, M. (1998). Phenotypic Evolution: A Reaction Norm Perspective . Sunderland: Sinauer Associates Inc.

Schlichting, C. D., \& Smith, H. (2002). Phenotypic plasticity: linking molecular mechanisms with evolutionary outcomes. Evolutionary Ecology, 16 (3), 189-211.

Schlosser, G., \& Wagner, G. P. (2004). Modularity in development and evolution . Chicago: The University of Chicago Press.

Siegal, M. L., \& Bergman, A. (2002). Waddington's canalization revisited: developmental stability and evolution. Proceedings of the National Academy of Sciences of the USA, 99 , 10528-10532.

Stearns, S. C., Kaiscr, M., \& Kawecki, T. J. (1995). The differential genetic and environmental canalization of fitness components in Drosophila melanogaster. Journal of Evolutionary Biolology, 8 , 539-557.

Stearns, S. C., \& Kawecki, T. J. (1994). Fitness sensitivity and the canalization of life-history traits.Evolution, $48,1438-1450$.

Teder, T., Tammaru, T., \& Esperk, T. (2008). Dependence of phenotypic variance in body size on environmental quality. American Naturalist, 172 , 223-232. 
Valladares, F., Balaguer, L., Martinez-Ferri, E., Perez-Corona, E., \& Manrique, E. (2002). Plasticity, instability and canalization: is the phenotypic variation in seedlings of sclerophyll oaks consistent with the environmental unpredictability of Mediterranean ecosystems? New Phytologist, 156 , 457-467.

Valladares, F., Gianoli, E., \& Gómez, J. M. (2007). Ecological limits to plant phenotypic plasticity.New Phytologist, 176 (4), 749-763.

Valladares, F., Sanchez-Gomez, D., \& Zavala, M. A. (2006). Quantitative estimation of phenotypic plasticity: Bridging the gap between the evolutionary concept and its ecological applications. Journal of Ecology, 94 (6), 1103-1116.

Waddington, C. H. (1941). Evolution of developmental systems. Nature, 147, 108-110.

Waddington, C. H. (1952). Selection of the genetic basis for an acquired character. Nature, 169, 625-626.

Waddington, C. H. (1956). Genetic assimilation of the bithorax phenotype. Evolution, 10 , 1-13.

Waddington, C. H. (1957). The strategy of the genes . London: George Allen \& Unwin Ltd.

Wagner, G. P., Booth, G., \& Bagheri-Chaichian, H. (1997). A population genetic theory of canalization. Evolution, 51 , 329-347.

Waitt, D. E., \& Levin, D. A. (1993). Phenotypic integration and plastic correlations in Phlox drumondii (Polemoniaceae). American Journal of Botany, 80 , 1224-1233.

Wang, S., Li, L., \& Zhou, D.-W. (2017). Morphological plasticity in response to population density varies with soil conditions and growth stage in Abutilon theophrasti (Malvaceae). Plant Ecology, 218 , 785-797.

Waxman, D., \& Peck, J. R. (1998). Pleiotropy and the preservation of perfection. Science, 279 , 1210-1213.

West-Eberhard, M. J. (2003).Developmental plasticity and evolution . Oxford: Oxford University Press.

White, J. (1979). The plant as a metapopulation. Annual Review of Ecology and Systematics, 10 , 109-145.

Wilkins, A. S. (1997). Canalization: a molecular genetic perspective. BioEssays, 19 , 257-262.

Woods, R. E., Sgro, C. M., Hercus, M. J., \& Hoffmann, A. A. (1999). The association between fluctuating asymmetry, trait variability, trait heritability, and stress: a multiply replicated experiment on combined stresses in Drosophila melanogaster . Evolution, 53 , 493-505.

Wylde, Z., \& Bonduriansky, R. (2020). Condition dependence of phenotypic integration and the evolvability of genitalic traits in a neriid fly. Biology Letters, 16 (5), 20200124.

Table 1 All traits and trait variables with their abbreviations and transformations in this study.

\begin{tabular}{llll}
\hline Trait/variable & Unit & Abbreviation & Transformation \\
\hline Total mass & $\mathrm{g}$ & TM & Log \\
Root mass ratio & $/$ & RMR & Log \\
Stem mass ratio & $/$ & SMR & Log \\
Petiole mass ratio & $/$ & PMR & Log \\
Lamina mass ratio & $/$ & LAMR & Log \\
Reproductive mass & $/$ & REMR & Log \\
ratio & & & \\
Stem length & $\mathrm{cm}$ & SL & Log \\
Stem diameter & $\mathrm{mm}$ & SD & Log \\
Main root length & $\mathrm{cm}$ & MRL & Log \\
Main root diameter & $\mathrm{mm}$ & MRD & Log \\
Lateral root length & $\mathrm{cm}$ & LRL & Log \\
Lateral root number & $/$ & LRN & Log
\end{tabular}




\begin{tabular}{llll}
\hline Trait/variable & Unit & Abbreviation & Transformation \\
\hline Petiole length & $\mathrm{cm}$ & $\mathrm{PL}$ & Log \\
Petiole angle & $\mathrm{o}$ & $\mathrm{PA}$ & $\mathrm{Sqrt}$ \\
The number of leaves & $/$ & $\mathrm{LN}$ & $\mathrm{Log}$ \\
Lamina size & $\mathrm{mm}$ & $\mathrm{LS}$ & $\mathrm{Log}$ \\
Coefficient of variation & $/$ & $\mathrm{CV}$ & $\mathrm{Log}$ \\
$\begin{array}{l}\text { Coefficient of } \\
\text { integration }\end{array}$ & $/$ & $\mathrm{CI}$ & $/$ \\
Number of significant & $/$ & $\mathrm{NC}$ & $\mathrm{Log}$ \\
correlations & & & $/$ \\
Relative plasticity & $/$ & REL RDPI & $/$ \\
Absolute plasticity & $/$ & ABS RDPI & \\
\hline
\end{tabular}

Table 2 Three-way ANOVAs on effects of growth stage (GS), soil condition (SC), population density (PD) and their interactions for all variables. Abbreviations for all variables are in Table 1.

\begin{tabular}{|c|c|c|c|c|c|c|c|c|}
\hline $\begin{array}{l}\text { Source of } \\
\text { Variation }\end{array}$ & $\begin{array}{l}\mathrm{REL} \\
\mathrm{RDPI}_{\mathrm{s}}\end{array}$ & $\begin{array}{l}\mathrm{REL} \\
\mathrm{RDPI}_{\mathrm{s}}\end{array}$ & $\begin{array}{l}\mathrm{ABS} \\
\mathrm{RDPI}_{\mathrm{S}}\end{array}$ & $\begin{array}{l}\mathrm{ABS} \\
\mathrm{RDPI}_{\mathrm{S}}\end{array}$ & $\log (\mathrm{NC})$ & $\log (\mathrm{NC})$ & $\log (\mathrm{CV})$ & $\log (\mathrm{C}$ \\
\hline & $F$ & $P$ & $F$ & $P$ & $F$ & $P$ & $F$ & $P$ \\
\hline GS & 0.08 & 0.799 & 0.95 & 0.337 & 0.21 & 0.645 & 0.00 & 0.957 \\
\hline $\mathrm{SC}$ & 0.07 & 0.775 & 0.39 & 0.539 & 0.58 & 0.448 & 0.26 & 0.612 \\
\hline $\mathrm{PD}$ & 2.44 & 0.129 & 5.26 & 0.029 & 3.40 & 0.019 & 3.51 & 0.032 \\
\hline $\begin{array}{l}\text { GS } \times \\
\text { SC }\end{array}$ & 2.12 & 0.155 & 3.55 & 0.069 & 0.07 & 0.792 & 0.11 & 0.739 \\
\hline $\begin{array}{l}\mathrm{GS} \times \\
\mathrm{PD}\end{array}$ & 0.87 & 0.359 & 0.72 & 0.402 & 2.37 & 0.072 & 1.00 & 0.369 \\
\hline $\begin{array}{l}\mathrm{SC} \times \\
\mathrm{PD}\end{array}$ & 0.00 & 0.958 & 0.93 & 0.341 & 0.56 & 0.640 & 1.04 & 0.355 \\
\hline $\begin{array}{l}\mathrm{GS} \times \\
\mathrm{SC} \times \\
\mathrm{PD}\end{array}$ & 0.62 & 0.437 & 1.27 & 0.268 & 1.10 & 0.351 & 0.41 & 0.664 \\
\hline
\end{tabular}

Table $3 F$-values for three-way ANOVAs on correlations among variables, with soil condition (SC), growth stage (GS), population density (PD), plasticity type (PT) and their interactions as effects. Abbreviations for all variables are in Table 1. $P<0.10,{ }^{* *} P<0.05,{ }^{* * *} P<0.01$.

\begin{tabular}{lllll}
\hline Source of Variation & REL RDPI & REL RDPI & ABS RDPI & ABS RDPI \\
\hline & NC & CV & NC & CV \\
SC & 4.11 & 0.70 & $61.52^{* *}$ & $8.54^{*}$ \\
GS & 1.30 & $14.49^{*}$ & $23.90^{* *}$ & 0.62 \\
$\mathrm{PD}$ & 5.47 & $18.36^{* *}$ & $105.29^{* * *}$ & 2.10 \\
$\mathrm{PT}$ & 1.10 & $18.02^{*}$ & $191.06^{* * *}$ & 0.06 \\
$\mathrm{SC} \times \mathrm{GS}$ & 2.97 & $31.77^{* *}$ & $272.81^{* * *}$ & $54.98^{* *}$ \\
$\mathrm{SC} \times \mathrm{PD}$ & 0.42 & $35.24^{* *}$ & $173.68^{* * *}$ & 2.84 \\
$\mathrm{SC} \times \mathrm{PY}$ & 3.32 & $50.33^{* *}$ & $270.46^{* * *}$ & $14.06^{*}$ \\
$\mathrm{GS} \times \mathrm{PD}$ & 0.56 & 1.92 & 4.05 & 2.47 \\
$\mathrm{GS} \times \mathrm{PT}$ & 0.71 & 0.56 & $33.92^{* *}$ & $8.88^{*}$ \\
$\mathrm{PD} \times \mathrm{PT}$ & 0.36 & $6.71^{*}$ & 4.90 & 1.70 \\
& & & &
\end{tabular}




\begin{tabular}{lllll}
\hline Source of Variation & REL RDPI & REL RDPI $_{\mathrm{s}}$ & $\mathrm{ABS} \mathrm{RDPI}_{\mathrm{s}}$ & $\mathrm{ABS} \mathrm{RDPI}_{\mathrm{s}}$ \\
\hline $\mathrm{SC} \times \mathrm{GS} \times \mathrm{PD}$ & 0.32 & $7.97^{*}$ & $75.35^{* * *}$ & 3.44 \\
$\mathrm{SC} \times \mathrm{GS} \times \mathrm{PT}$ & 0.01 & 0.06 & $82.38^{* * *}$ & 1.17 \\
$\mathrm{SC} \times \mathrm{PD} \times \mathrm{PT}$ & 0.02 & 2.41 & $26.25^{* *}$ & 1.46 \\
$\mathrm{GS} \times \mathrm{PD} \times \mathrm{PT}$ & 0.55 & 2.39 & $12.22^{*}$ & 0.85 \\
\hline
\end{tabular}

Table 4 General Linear Model (GLM) analyses on regressive relationships of relative plasticity (REL RDPI $)$ and the degree of plasticity (absolute plasticity, $\mathrm{ABS} \mathrm{RDPI}_{\mathrm{s}}$ ) with the number of significant correlations (NC) among traits and coefficient of variations of traits (CV) in response to density under infertile and fertile soil conditions at $50 \mathrm{~d}$ and $70 \mathrm{~d}$ of plant growth. Abbreviations for all variables are in Table $1 . P<0.10,{ }^{* *} P$ $<0.05,{ }^{* * *} P<0.01$.

\begin{tabular}{lllllll}
\hline Plasticity & Stage & Soil & NC & NC & CV & CV \\
\hline & & & $F_{\text {SloPe }}$ & $F_{\text {IntercePt }}$ & $F_{\text {SloPe }}$ & $F_{\text {IntercePt }}$ \\
REL RDPI $_{\mathrm{s}}$ & $50 \mathrm{D}$ & Infertile & 0.41 & 0.70 & 0.92 & 0.94 \\
& & Fertile & $\mathbf{1 1 . 9 7 * * *}$ & 0.69 & 1.62 & 0.61 \\
& \multirow{2}{*}{ 70D } & Infertile & 1.39 & $2.17^{*}$ & $\mathbf{2 . 4 8}$ & 0.61 \\
& & Fertile & $\mathbf{6 . 3 0}^{* * *}$ & $4.18^{* * *}$ & $\mathbf{3 . 9 2}^{* *}$ & 0.85 \\
ABS RDPI $_{\mathrm{s}}$ & $50 \mathrm{D}$ & Infertile & 0.92 & 0.69 & 0.44 & 0.36 \\
& & Fertile & $\mathbf{6 . 2 9}^{* * *}$ & 1.35 & $\mathbf{2 . 5 9 *}$ & 1.22 \\
& \multirow{2}{*}{ 70D } & Infertile & 0.56 & 0.61 & 0.07 & $2.56^{*}$ \\
& & Fertile & $\mathbf{5 . 9 3}^{* * *}$ & $4.61^{* * *}$ & $\mathbf{1 4 . 1 4}^{* * *}$ & 1.40 \\
\hline
\end{tabular}

\section{Figure legends}

Fig. 1 Mean coefficient of variation (CV), coefficient of integration (CI) and mean number of significant correlations with other traits $(\mathrm{NC})$, and mean relative plasticity $\left(\mathrm{REL} R D P I_{\mathrm{S}}\right.$ ) and absolute plasticity $(\mathrm{ABS}$ $\mathrm{RDPI}_{\mathrm{s}}$ ) for all traits in response to density under infertile and fertile soil conditions at $50 \mathrm{~d}$ and $70 \mathrm{~d}$. Different letters and $P$ values denote significant differences between densities within each soil conditions and across both soil conditions respectively $(P<0.05)$.

Fig. 2 Regressions on the degree of plasticity $\left(\mathrm{ABS} R D P I_{\mathrm{s}}\right)$ vs. trait canalization $(\mathrm{CV})$ and integration (NC) at different densities for plants under infertile and fertile soil conditions at $50 \mathrm{~d}$ and $70 \mathrm{~d}$ of growth. H-L: high-low density plasticity, M-L: medium-low density plasticity.

Fig. 1 Mean coefficient of variation (CV), coefficient of integration (CI) and mean number of significant correlations with other traits $(\mathrm{NC})$, and mean relative plasticity $\left(\mathrm{REL} R \mathrm{RPI} \mathrm{I}_{\mathrm{s}}\right.$ ) and absolute plasticity $(\mathrm{ABS}$ $\mathrm{RDPI}_{\mathrm{S}}$ ) for all traits in response to density under infertile and fertile soil conditions at $50 \mathrm{~d}$ and $70 \mathrm{~d}$. Different letters and $P$ values denote significant differences between densities within each soil conditions and across both soil conditions respectively $(P<0.05)$.

Fig. 2 Regressions on the degree of plasticity $\left(A B S R^{2} I_{s}\right)$ vs. trait canalization $(C V)$ and integration (NC) at different densities for plants under infertile and fertile soil conditions at $50 \mathrm{~d}$ and $70 \mathrm{~d}$ of growth. H-L: high-low density plasticity, M-L: medium-low density plasticity. 